\title{
Wall crossing for derived categories of moduli spaces of sheaves on rational surfaces
}

\author{
Matthew Robert Ballard
}

\begin{abstract}
We remove the global quotient presentation input in the theory of windows in derived categories of smooth Artin stacks of finite type. It is replaced by a condition asking for the existence of a special atlas along the removed substack, which proves a bit easier to deal with in practice. As an application, we use existing results on the flipping of strata for the wall crossing of Gieseker semi-stable torsion-free sheaves of rank two on rational surfaces to produce semi-orthogonal decompositions relating the different moduli stacks. The complementary pieces of these semi-orthogonal decompositions are derived categories of products of Hilbert schemes of points on the surface.
\end{abstract}

\section{Introduction}

A central question in the theory of derived categories is the following: given a smooth, projective variety $X$, how does one find interesting semi-orthogonal decompositions of its derived category, $\mathrm{D}^{\mathrm{b}}(\operatorname{coh} X)$ ? Historically, two different parts of algebraic geometry have fed this question: birational geometry and moduli theory. The references [Or193, BO95, Or197, Bri02, Kaw02a, Kaw06, Kuz10] provide a non-exhaustive highlight reel for this approach.

This paper focuses on the intersection of birational geometry and moduli theory. Namely, given some moduli problem equipped with a notion of stability, variation of the stability condition often leads to birational moduli spaces. As such, it is natural to compare the derived categories in this situation. Let us consider the well-understood situation of torsion-free rank two semistable sheaves on rational surfaces [EG95, FQ95, MW97]. The flipping of unstable strata under change of polarization was investigated to understand the change in the Donaldson invariants. It provides the input for the following result, which can be viewed as a categorification of the wall-crossing formula for Donaldson invariants.

ThEOREM 1 (Corollary 3.10). Let $S$ be a smooth rational surface over $\mathbb{C}$ with $K_{S}<0$, and let $L_{-}$and $L_{+}$be ample lines bundles on $S$ separated by a single wall defined by unique divisor $\xi$ satisfying

$$
\begin{gathered}
L_{-} \cdot \xi<0<L_{+} \cdot \xi, \\
0 \leqslant \omega_{S}^{-1} \cdot \xi .
\end{gathered}
$$

Received 13 January 2016, accepted in final form 23 July 2016.

2010 Mathematics Subject Classification 14F05.

Keywords: moduli spaces, derived categories, variation of stability.

This journal is (C) Foundation Compositio Mathematica 2017. This article is distributed with Open Access under the terms of the Creative Commons Attribution Non-Commercial License, which permits non-commercial reuse, distribution, and reproduction in any medium, provided that the original work is properly cited. For commercial re-use, please contact the Foundation Compositio Mathematica.

The author was supported by a Simons Collaboration Grant and NSF Standard Grant DMS-1501813. 


\section{R. BALLARD}

Let $\mathcal{M}_{L_{ \pm}}(\Delta, c)$ be the $\mathbb{G}_{m}$-rigidified moduli stack of Gieseker $L_{ \pm}$-semi-stable torsion-free sheaves of rank two with first Chern class $c_{1}$ and second Chern class $c_{2}$.

There is a semi-orthogonal decomposition of $\mathrm{D}^{\mathrm{b}}\left(\operatorname{coh} \mathcal{M}_{L_{+}}(\Delta, c)\right)$ as

$$
\langle\underbrace{\mathrm{D}^{\mathrm{b}}\left(\operatorname{coh} H^{l_{\xi}}\right), \ldots, \mathrm{D}^{\mathrm{b}}\left(\operatorname{coh} H^{l_{\xi}}\right)}_{\mu_{\xi}}, \ldots, \underbrace{\mathrm{D}^{\mathrm{b}}\left(\operatorname{coh} H^{0}\right), \ldots, \mathrm{D}^{\mathrm{b}}\left(\operatorname{coh} H^{0}\right)}_{\mu_{\xi}}, \mathrm{D}^{\mathrm{b}}\left(\operatorname{coh} \mathcal{M}_{L_{-}}(\Delta, c)\right)\rangle,
$$

where

$$
l_{\xi}:=\left(4 c_{2}-c_{1}^{2}+\xi^{2}\right) / 4, \quad H^{l}:=\operatorname{Hilb}^{l}(S) \times \operatorname{Hilb}^{l_{\xi}-l}(S), \quad \mu_{\xi}:=\omega_{S}^{-1} \cdot \xi,
$$

with the convention that $\operatorname{Hilb}^{0}(S):=\operatorname{Spec} \mathbb{C}$.

While Theorem 1 is interesting in its own right, the method might be more so. Indeed, Theorem 1 represents one of multiple possible applications, including to moduli spaces of Bridgeland semi-stable objects on rational surfaces; see [ABCH13].

Theorem 1 follows from the general technology that goes under the heading of windows in derived categories. Windows provide a framework for addressing the central question put forth above; they are a machine for manufacturing interesting semi-orthogonal decompositions of $\mathrm{D}^{\mathrm{b}}(\operatorname{coh} X)$. They have a rich history with contributions by many mathematicians and physicists [Kaw02b, vdBer04, Or109, HHP08, Seg11, HW12, Shi12, Hal15a, BFK16, DS14]. However, windows have not yet achieved their final form. Previous work dealt with an Artin stack $\mathcal{X}$ plus a choice of global quotient presentation $\mathcal{X}=[X / G]$. Locating an appropriate quotient presentation for a given $\mathcal{X}$ is not convenient in applications, in particular the above, so one would like a definition of a window more intrinsic to $\mathcal{X}$. Section 2 provides such a definition using an appropriate type of groupoid in Białynicki-Birula strata and extends the prior results on semi-orthogonal decompositions [BFK16] to this setting; see Theorem 2.29. The method demonstrates that the global structure of $\mathcal{X}$ is not of upmost importance. Indeed, it is only the structure of the closed substack $\mathcal{Z}$ marked for removal and the structure of $\mathcal{X}$ along $\mathcal{Z}$ that one needs to use get these results.

A similar extension appeared in [Hal15b].

\section{Semi-orthogonal decompositions and BB strata}

For the whole of this section, $k$ will denote an algebraically closed field. The term variety means a separated, reduced scheme of finite type over $k$. All points of a variety are closed points unless explicitly stated otherwise.

In this section, we extend the results of [BFK16] by removing the global quotient presentation from the input data. We try to keep this section as self-contained as possible.

\subsection{Truncations of sheaves on BB strata}

We begin with the following definition. Let $X$ be a smooth quasi-projective variety equipped with a $\left(\mathbb{G}_{m}\right)$-action $\sigma: X \times \mathbb{G}_{m} \rightarrow X$. Let $X^{\mathbb{G}_{m}}$ denote the fixed subscheme of the action, and let $X_{f}$ be a choice of a connected component of the fixed locus. We recall the following well-known result of Białynicki-Birula.

Theorem 2.1 ([Bia73, Theorems 2.1 and 4.1]). The fixed locus $X^{\mathbb{G}_{m}}$ is smooth and is a closed subvariety of $X$. Let $X_{f}$ be a connected component of $X^{\mathbb{G}_{m}}$. There exist a unique smooth and 


\section{WALL CROSSING FOR DERIVED CATEGORIES}

locally closed $\mathbb{G}_{m}$-invariant subvariety $X^{+}$of $X$ and a unique morphism $\pi: X^{+} \rightarrow X_{f}$ such that

a) $X_{f}$ is a closed subvariety of $X^{+}$;

b) the morphism $\pi: X^{+} \rightarrow X_{f}$ is an equivariantly locally-trivial fibration of affine spaces over $X_{f}$;

c) for a point $x \in X_{f}$, there is an equality

$$
T_{x} X^{+}=\left(T_{x} X\right)^{\geqslant 0},
$$

where the right-hand side is the subspace of non-negative weights of the geometric tangent space.

Remark 2.2. Note that the weights on the affine fibers are all positive and the set of closed points of a $X^{+}$from Theorem 2.1 is

$$
\left\{x \in X \mid \lim _{\alpha \rightarrow 0} \sigma(\alpha, x) \in X_{f}\right\},
$$

so one may think of $X^{+}$as the set of points that flow into $X_{f}$ as $\alpha \rightarrow 0$.

DeFinition 2.3. Let $X$ be a smooth quasi-projective variety equipped with a $\mathbb{G}_{m}$-action. Let $X_{f}$ be a choice of a connected component of $X^{\mathbb{G}_{m}}$. The $B B$ stratum associated with $X_{f}$ is the variety $X^{+}$appearing in Theorem 2.1.

Similarly, we have the following definition.

Definition 2.4. If $X=X^{+}$, then we shall also say that $X$ is a BB stratum. In particular, we require that $X^{\mathbb{G}_{m}}=X_{f}$ is connected in this situation.

Remark 2.5. Note that $X$ being a BB stratum is equivalent to the existence of an action map $\mathbb{A}^{1} \times X \rightarrow X$ with $X^{\mathbb{G}_{m}}$ connected. Here we view $\mathbb{A}^{1}$ as a monoidal scheme via multiplication.

Let $X$ be a BB stratum. Let us consider the local situation first. So $X=\operatorname{Spec} R\left[x_{1}, \ldots, x_{n}\right]$ and we have a coaction map, also denoted by $\sigma$,

$$
\sigma: R\left[x_{1}, \ldots, x_{n}\right] \rightarrow R\left[x_{1}, \ldots, x_{n}, u, u^{-1}\right],
$$

where the weights of the $x_{i}$ are positive. The fixed locus is then $X_{f}=\operatorname{Spec} R$. Let $M$ be a $\mathbb{G}_{m}$-equivariant module over $R$, that is, a quasi-coherent $\mathbb{G}_{m}$-equivariant sheaf on $X_{f}$. Then we have a map

$$
\Delta: M \rightarrow M\left[u, u^{-1}\right]
$$

corresponding to the equivariant structure. One sets

$$
M_{i}:=\left\{m \in M \mid \Delta(m)=m \otimes u^{i}\right\} .
$$

If we try to globalize this construction, then two different $M_{i}$ are identified under an automorphism of $M$, which has degree zero with respect to $\mathbb{G}_{m}$. Thus, for any quasi-coherent $\mathbb{G}_{m^{-}}$ equivariant sheaf, this gives a quasi-coherent $\mathbb{G}_{m}$-quasi-coherent sheaf $\mathcal{E}_{i}$ on $X_{f}$.

Lemma 2.6. Let $X$ have an action of $\mathbb{G}_{m}$, and let $\mathcal{E}$ be a coherent $\mathbb{G}_{m}$-equivariant sheaf on the fixed locus $X_{f}$. Then there is a functorial decomposition

$$
\mathcal{E} \cong \bigoplus_{i \in \mathbb{Z}} \mathcal{E}_{i}
$$

In particular, for each $i \in \mathbb{Z}$, the functor $\mathcal{E} \mapsto \mathcal{E}_{i}$ is exact. 


\section{R. BALlard}

Proof. This is standard.

Corollary 2.7. Let $X$ have an action of $\mathbb{G}_{m}$, and let $\mathcal{E}$ be a bounded complex of coherent $\mathbb{G}_{m}$-equivariant sheaves on the fixed locus $X_{f}$. Then there is a functorial decomposition of the complex

$$
\mathcal{E} \cong \bigoplus_{i \in \mathbb{Z}} \mathcal{E}_{i}
$$

This descends to the derived category $\mathrm{D}^{\mathrm{b}}\left(\operatorname{coh}\left[X_{f} / \mathbb{G}_{m}\right]\right)$.

Proof. This follows immediately from Lemma 2.6.

Definition 2.8. For a subset $I \subseteq \mathbb{Z}$, we say that a complex $\mathcal{E}$ from $\mathrm{D}^{\mathrm{b}}\left(\operatorname{coh}\left[X_{f} / \mathbb{G}_{m}\right]\right)$ has weights concentrated in $I$ if $\left(\mathcal{H}^{p}(\mathcal{E})\right)_{i}=0$ for all $p \in \mathbb{Z}$ and $i \notin I$.

Now, we turn our attention to $\mathbb{G}_{m}$-equivariant sheaves on $X$ itself. Let $j: X_{f} \rightarrow X$ be the inclusion.

DeFinition 2.9. Let $\mathcal{E}$ be an object of $\mathrm{D}^{\mathrm{b}}\left(\operatorname{coh}\left[X / \mathbb{G}_{m}\right]\right)$, and let $I \subseteq \mathbb{Z}$. We say that $\mathcal{E}$ has weights concentrated in $I$ if $\mathbf{L} j^{*} \mathcal{E}$ has weights concentrated in $I$.

Next, we want to give a procedure to truncate the weights. We first again go back to the local case with $X=\operatorname{Spec} R\left[x_{1}, \ldots, x_{n}\right]$, where the $x_{i}$ have positive weight. Let $M$ be a $\mathbb{G}_{m}$-equivariant module over $R\left[x_{1}, \ldots, x_{n}\right]$. We can still consider

$$
M_{a}:=\left\{m \in M \mid \sigma(m)=m \otimes u^{a}\right\} .
$$

However, this is no longer a submodule of $M$ as multiplication by $x_{i}$ will raise the weight. But,

$$
M_{\geqslant a}:=\bigoplus_{j \geqslant a} M_{j}
$$

is a submodule of $M$ and inherits a natural $\mathbb{G}_{m}$-equivariant structure. The assignment $M \mapsto M_{\geqslant a}$ is functorial with respect to $\mathbb{G}_{m}$-equivariant morphisms, so gives an exact functor

$$
\tau_{\geqslant a}: \operatorname{coh}\left[X / \mathbb{G}_{m}\right] \rightarrow \operatorname{coh}\left[X / \mathbb{G}_{m}\right],
$$

which, of course, descends to the derived category

$$
\tau_{\geqslant a}: \mathrm{D}^{\mathrm{b}}\left(\operatorname{coh}\left[X / \mathbb{G}_{m}\right]\right) \rightarrow \mathrm{D}^{\mathrm{b}}\left(\operatorname{coh}\left[X / \mathbb{G}_{m}\right]\right) .
$$

Now, let us consider the global situation. Since we have a $\mathbb{G}_{m}$-invariant cover of $X$ of the form Spec $R\left[x_{1}, \ldots, x_{n}\right]$, where the $x_{i}$ have positive weights and $R$ has weight zero, we can glue this construction to get

$$
\begin{aligned}
\tau_{\geqslant a}: \mathrm{D}^{\mathrm{b}}\left(\operatorname{coh}\left[X / \mathbb{G}_{m}\right]\right) & \rightarrow \mathrm{D}^{\mathrm{b}}\left(\operatorname{coh}\left[X / \mathbb{G}_{m}\right]\right), \\
\mathcal{E} & \mapsto \mathcal{E}_{\geqslant a} .
\end{aligned}
$$

Definition 2.10. Let $a \in \mathbb{Z}$. For a bounded complex $\mathcal{E}$ of coherent $\mathbb{G}_{m}$-equivariant sheaves on $X$, one calls $\mathcal{E}_{\geqslant a}$ a weight truncation of $\mathcal{E}$.

Lemma 2.11. If $\mathcal{E}$ has weights concentrated in $I$, then $\tau_{\geqslant a} \mathcal{E}$ has weights concentrated in $I \cap[a, \infty)$. Moreover, if $\mathcal{E}$ has weights concentrated in $[a, \infty)$, then there is a natural quasi-isomorphism $\mathcal{E} \cong \mathcal{E}_{\geqslant a}$. 


\section{WALL CROSSING FOR DERIVED CATEGORIES}

Proof. We can check this computation locally and assume that $\mathcal{E}$ is a bounded complex of locally free sheaves. Then, one sees that there is a natural isomorphism

$$
\left.\left(\mathcal{E}_{\geqslant a}\right)\right|_{X_{f}} \cong\left(\left.\mathcal{E}\right|_{X_{f}}\right)_{\geqslant a}
$$

Looking at the left-hand side, we see that the complex has weights in $[a, \infty) \cap I$.

Now, we level up and consider an appropriate type of groupoid in BB strata. Let $X^{1} \underset{t}{\stackrel{s}{\rightrightarrows}} X^{0}$ be a groupoid scheme with $s, t$ smooth and $X^{1}, X^{0}$ smooth and quasi-projective. In general, we shall suppress the additional data packaged in a groupoid scheme, as we do in the following statement. Assume that we have a commutative diagram

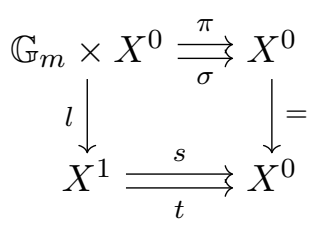

of groupoid schemes with $l$ a closed embedding and $\sigma$ an action. Then, we can define a morphism

$$
\begin{aligned}
A: \mathbb{G}_{m} \times X^{1} & \rightarrow X^{1}, \\
\left(\alpha, x_{1}\right) & \mapsto l\left(\alpha, t\left(x_{1}\right)\right) \cdot x_{1} \cdot l\left(\alpha^{-1}, \sigma\left(\alpha, s\left(x_{1}\right)\right)\right),
\end{aligned}
$$

where the central dot is the notation for the multiplication $m: X^{1} \times_{s, X^{0}, t} X^{1} \rightarrow X^{1}$.

Lemma 2.12. The morphism $A$ defines an action of $\mathbb{G}_{m}$ on $X^{1}$ making both $s$ and $t$ equivariant.

Proof. This is straightforward to verify, so the details are left out.

Definition 2.13. If we have a commutative diagram of groupoids as above, the action $A$ is the called the adjoint of $\mathbb{G}_{m}$ on $X^{1}$. We say that a groupoid scheme $X^{\bullet}$ is a stacky BB stratum if $A$ extends to a morphism $\mathbb{A}^{1} \times X^{1} \rightarrow X^{1}$ and $\left(X^{0}\right)^{\mathbb{G}_{m}}$ is connected. Similarly, we say that the associated stack $\left[X^{0} / X^{1}\right]$ is a stacky BB stratum if $X^{\bullet}$ is one.

We can pass to the fixed loci of the $\mathbb{G}_{m}$-actions on $X^{\bullet}$ to get another groupoid scheme $\left(X^{1}\right)^{\mathbb{G}_{m}} \rightrightarrows\left(X^{0}\right)^{\mathbb{G}_{m}}$ which we call the fixed substack and denote by $\left[X^{0} / X^{1}\right]_{f}$. It is straightforward to check that $\left(X^{1}\right)^{\mathbb{G}_{m}} \rightrightarrows\left(X^{0}\right)^{\mathbb{G}_{m}}$ is also a stacky BB stratum.

By taking the limit as $\alpha \rightarrow 0$ in $\mathbb{G}_{m}$, we get an induced projection that we denote by $\pi:\left[X^{0} / X^{1}\right] \rightarrow\left[X^{0} / X^{1}\right]_{0}$.

Remark 2.14. Note that if $A$ extends to a morphism $\mathbb{A}^{1} \times X^{1} \rightarrow X^{1}$, then $X^{1}$ and $X^{0}$ are unions of BB strata. For simplicity of exposition, we require the connectedness of $\left(X^{0}\right)^{\mathbb{G}_{m}}$. The disconnected case can be handled using the same arguments with minor modifications.

Remark 2.15. The language of the definition is a bit misleading, as the careful reader will note that it is not clear that the notion is intrinsic to $\mathcal{X}=\left[X^{0} / X^{1}\right]$ and does not depend on the presentation. It seems that this gives a connected component of the Hom-stack Hom $\left(\left[\mathbb{A}^{1} / \mathbb{G}_{m}\right], \mathcal{X}\right)$ as studied in [HP14]. However, in the application of interest and in many moduli problems, the data of a stacky BB stratum is easy to extract from a description as projective bundles over lower-dimensional moduli spaces. 


\section{R. BALLARD}

Lemma 2.16. Let $X^{\bullet}$ be a stacky $B B$ stratum, and let $\tau_{\geqslant u}$ be the truncation functor on $\mathrm{D}^{\mathrm{b}}\left(\operatorname{coh}\left[X^{0} / \mathbb{G}_{m}\right]\right)$ as previously defined. Then, $\tau_{\geqslant u}$ descends to an endofunctor

$$
\tau_{\geqslant u}: \mathrm{D}^{\mathrm{b}}\left(\operatorname{coh} X^{\bullet}\right) \rightarrow \mathrm{D}^{\mathrm{b}}\left(\operatorname{coh} X^{\bullet}\right) .
$$

Furthermore, the weight decomposition on $\mathrm{D}^{\mathrm{b}}\left(\operatorname{coh}\left[X_{0}^{0} / \mathbb{G}_{m}\right]\right)$ descends to $\mathrm{D}^{\mathrm{b}}\left(\operatorname{coh}\left[X_{0}^{0} / X_{0}^{1}\right]\right)$.

Proof. Recall that a quasi-coherent sheaf on $X^{\bullet}$ is a pair $(\mathcal{E}, \theta)$ with $\mathcal{E} \in \mathrm{Q} \operatorname{coh}\left(X^{0}\right)$ and $\theta: s^{*} \mathcal{E} \stackrel{\sim}{\rightarrow}$ $t^{*} \mathcal{E}$ an isomorphism satisfying an appropriate cocycle condition and identity condition. Note that any sheaf on $X^{\bullet}$ carries a $\mathbb{G}_{m}$-equivariant structure by pulling back along $l$, so truncation is well defined on $\mathcal{E}$. For any coherent sheaf $\mathcal{E}$ on $X^{\bullet}$, we will show that

$$
\theta\left(t^{*}\left(\tau_{\geqslant u} \mathcal{E}\right)\right) \subset s^{*}\left(\tau_{\geqslant u} \mathcal{E}\right)
$$

This suffices to show that $\tau_{\geqslant u}$ descends as $\theta^{-1}=i^{*} \theta$, where $i: X^{1} \rightarrow X^{1}$ is the inverse over $X^{0}$.

This question is local, so we may assume $X^{1}=\operatorname{Spec} S$ and $X^{0}=\operatorname{Spec} R$. Let $M$ be the module corresponding to $\mathcal{E}$, so that $\theta: M \otimes_{R, t} S \rightarrow M \otimes_{R, s} S$ is an isomorphism. As we will need to use it, we now recall the cocycle condition in this local situation. We have a diagram

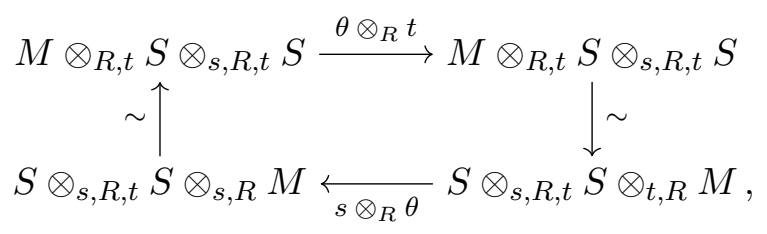

which gives us an isomorphism

$$
M \otimes_{R, t} S \otimes_{s, R, t} S \stackrel{\left(s \otimes_{R} \theta\right) \circ\left(\theta \otimes_{R} t\right)}{\longrightarrow} M \otimes_{R, t} S \otimes_{s, R, t} S .
$$

We can also tensor $\theta$ over $S$ with $m: S \rightarrow S \otimes_{s, R, t} S$ to get another isomorphism. The cocycle condition is

$$
\left(s \otimes_{R} \theta\right) \circ\left(\theta \otimes_{R} t\right)=\theta \otimes_{S} m .
$$

Now, we wish to check that $\theta\left(M_{j} \otimes_{R} S\right) \subset M_{\geqslant j} \otimes_{R} S$. We do this as follows. First, since $A: S \rightarrow S\left[u, u^{-1}\right]$ has image in $S[u]$, the image of

$$
(l \otimes 1 \otimes l) \circ(m \otimes 1) \circ m:=\tilde{A}: S \rightarrow R\left[u_{1}, u_{1}^{-1}\right] \otimes_{\pi, R, t} S \otimes_{s, R, \sigma} R\left[u_{2}, u_{2}^{-1}\right]
$$

must lie in the $S$-subalgebra generated by the $u_{1}^{i} u_{2}^{j}$ with $i-j \geqslant 0$. Since $\theta$ satisfies the cocyle condition, we can factor $\theta \otimes_{S} \tilde{A}$ as

$$
\theta \otimes_{S} \tilde{A}=\left(1 \otimes_{R} \theta \otimes_{R} 1\right) \circ\left(\bar{\theta} \otimes_{R} 1 \otimes_{R} 1\right) \circ\left(1 \otimes_{R} \theta \otimes_{R} 1\right) \circ\left(1 \otimes_{R} 1 \otimes_{R} \bar{\theta}\right) .
$$

Applying this to $m_{j} \in M_{j}$, we have

$$
\left.\left(\theta \otimes_{S} \tilde{A}\right)\left(m_{j}\right)=\sum_{i} \theta\left(\theta\left(m_{j}\right)_{i}\right)\right) u_{1}^{i} u_{2}^{j},
$$

where

$$
\theta\left(m_{j}\right)=\sum_{i} \theta\left(m_{j}\right)_{i} u_{1}^{i}
$$

As $i-j \geqslant 0$, we see that the $\theta\left(m_{j}\right)$ are elements of $M_{\geqslant j} \otimes_{R, s} S$ and $\theta$ preserves the truncation.

For the final statement, repeating the previous argument and assuming that $\mathbb{G}_{m}$ acts trivially on $R$ and $S$ shows that $\theta$ preserves the whole splitting via weights. 


\section{WALL CROSSING FOR DERIVED CATEGORIES}

EXAMPLE 2.17. Let $G$ be a linear algebraic group acting on a variety $X$. Assume that we have a one-parameter subgroup $\lambda: \mathbb{G}_{m} \rightarrow G$ and a connected component $Z_{\lambda}^{0}$ of the fixed locus. Then we have the BB stratum $Z_{\lambda}$ and its orbit $S_{\lambda}:=G \cdot Z_{\lambda}$. Let

$$
P(\lambda):=\left\{g \in G \mid \lim _{\alpha \rightarrow 0} \lambda(\alpha) g \lambda(\alpha)^{-1} \text { exists }\right\} .
$$

There is an induced action of $P(\lambda)$ on $Z_{\lambda}$. In general, $\stackrel{P}{P} \stackrel{P}{\times} Z_{\lambda}$ is a resolution of singularities of $S_{\lambda}$. If we assume that this map is an isomorphism, then we get what is called an elementary stratum in the language of [BFK16]. Since

$$
\left[G \stackrel{P(\lambda)}{\times} Z_{\lambda} / G\right] \cong\left[Z_{\lambda} / P(\lambda)\right]
$$

and the groupoid

$$
P(\lambda) \times Z_{\lambda} \underset{\sigma}{\stackrel{\pi}{\rightrightarrows}} Z_{\lambda}
$$

is a stacky BB stratum, we see that $\left[S_{\lambda} / G\right]$ is a stacky BB stratum. The simplest case is $G=\mathbb{G}_{m}$.

\subsection{Removing stacky BB strata and comparing derived categories}

Let $\mathcal{X}$ be a smooth Artin stack of finite type over $k$.

Definition 2.18. Let $i: \mathcal{Z} \rightarrow \mathcal{X}$ be a smooth closed substack that is also a stacky BB stratum, and let $l: \mathcal{Z}_{0} \rightarrow \mathcal{X}$ be the closed immersion of the fixed substack. Let $\mathcal{E}$ be a bounded complex of coherent sheaves on $\mathcal{X}$, and let $I \subseteq \mathbb{Z}$. We say that $\mathcal{E}$ has weights concentrated in $I$ if $\mathbf{L} l^{*} \mathcal{E} \in$ $\mathrm{D}^{\mathrm{b}}\left(\operatorname{coh} \mathcal{Z}_{0}\right)$ has weights concentrated in $I$.

Remark 2.19. It is useful to note that the condition of $\mathcal{Z}$ being a stacky BB stratum implies that its conormal complex is actually concentrated in a single degree.

Definition 2.20. Let $\mathcal{Z}$ be a stacky BB stratum in $\mathcal{X}$, and let $I \subseteq \mathbb{Z}$. The $I$-window associated with $\mathcal{Z}$ is the full subcategory whose objects have weights concentrated in $I$. We denote this subcategory by $\mathrm{W}^{\mathcal{F}}\left(I, \mathcal{Z}_{0}\right)$.

Lemma 2.21. Assume that the weights of the conormal sheaf of $\mathcal{Z}$ in $\mathcal{X}$ are all strictly negative, and let $t_{\mathcal{Z}}$ be the weight of the relative canonical sheaf $\omega_{\mathcal{Z} \mid \mathcal{X}}$. Set $\mathcal{U}:=\mathcal{X} \backslash \mathcal{Z}$, and let $j: \mathcal{U} \rightarrow \mathcal{X}$ be the inclusion. Then the functor

$$
j^{*}:{ }^{\mathrm{W}}=\left(I, \mathcal{Z}_{0}\right) \rightarrow \mathrm{D}^{\mathrm{b}}(\operatorname{coh} \mathcal{U})
$$

is fully faithful whenever $I$ is contained in a closed interval of length less than $-t_{\mathcal{Z}}$.

Proof. The argument here is essentially due to Teleman [Tel00, Section 2]. The author first learned of it in [Hal15a]. It amounts to descending through a few spectral sequences. For the convenience of the reader and to keep the paper self-contained, we recall it in some detail.

For any two objects $\mathcal{E}, \mathcal{F}$ of $\mathrm{D}^{\mathrm{b}}(\operatorname{coh} \mathcal{X})$, there is an exact triangle of graded vector spaces

$$
\operatorname{Hom}_{\mathcal{X}, \mathcal{Z}}(\mathcal{E}, \mathcal{F}) \rightarrow \operatorname{Hom}_{\mathcal{X}}(\mathcal{E}, \mathcal{F}) \stackrel{j^{*}}{\rightarrow} \operatorname{Hom}_{\mathcal{U}}\left(j^{*} \mathcal{E}, j^{*} \mathcal{F}\right)
$$

coming from applying the exact triangle of derived functors

$$
\mathbf{R} \Gamma_{\mathcal{Z}}(\mathcal{X},-) \rightarrow \mathbf{R} \Gamma(\mathcal{X},-) \stackrel{j^{*}}{\rightarrow} \mathbf{R} \Gamma(\mathcal{U},-)
$$

to $\mathbf{R} \mathcal{H} \operatorname{mom}_{\mathcal{X}}(\mathcal{E}, \mathcal{F})$. Therefore, a necessary and sufficient condition for the full faithfulness of $j^{*}$ is the vanishing of $\operatorname{Hom}_{\mathcal{X}, \mathcal{Z}}(\mathcal{E}, \mathcal{F})$. 


\section{R. BALLARD}

The exact triangle in equation (2.1) comes from applying $\mathbf{R} \Gamma(\mathcal{X},-)$ to the following exact triangle of functors:

$$
\mathcal{H}_{\mathcal{Z}} \rightarrow \mathrm{Id} \rightarrow \mathbf{R} j_{*} j^{*}
$$

Here, $\mathcal{H}_{\mathcal{Z}}$ is the derived sheafy local cohomology functor. The complex $\mathcal{H}_{\mathcal{Z}}(\mathcal{G})$ is not schemetheoretically supported on $\mathcal{Z}$, but there is a filtration bounded from above by powers of the ideal sheaf $\mathcal{I}_{\mathcal{Z}}$. The associated graded sheaves are scheme-theoretically supported on $\mathcal{Z}$. Furthermore, since $\mathcal{Z}$ is smooth, the $s$ th associated graded piece is isomorphic to $\mathbf{L} i^{*} \mathcal{G} \otimes \operatorname{Sym}^{s}\left(\mathcal{T}_{\mathcal{Z} \mid \mathcal{X}}\right) \otimes \omega_{\mathcal{Z} \mid \mathcal{X}}^{-1}$.

We may now take global sections on $\mathcal{Z}$ which we may factor through two pushforwards: one by $\pi: \mathcal{Z} \rightarrow \mathcal{Z}_{0}$ and one by the rigidification map $r: \mathcal{Z}_{0} \rightarrow \mathcal{Z}_{0}^{\mathbb{G}_{m}}$ [ACV03, Theorem 5.15]. The pushforward $r_{*}: \mathrm{D}^{\mathrm{b}}\left(\operatorname{coh} \mathcal{Z}_{0}\right) \rightarrow \mathrm{D}^{\mathrm{b}}\left(\operatorname{coh} \mathcal{Z}_{0}^{\mathbb{G}_{m}}\right)$ projects onto the weight zero component of the weight decomposition. Thus, to establish vanishing, it suffices to show that there is no weight zero component in the splitting of

$$
\pi_{*}\left(\mathbf{L} i^{*} \mathcal{G} \otimes \operatorname{Sym}^{s}\left(\mathcal{T}_{\mathcal{Z} \mid \mathcal{X}}\right) \otimes \omega_{\mathcal{Z} \mid \mathcal{X}}^{-1}\right) .
$$

Since we can resolve anything using pullbacks from $\mathcal{Z}_{0}$, we can use the projection formula to get

$$
\left.\left.\pi_{*}\left(\mathbf{L} i^{*} \mathcal{G} \otimes \operatorname{Sym}^{s}\left(\mathcal{T}_{\mathcal{Z} \mid \mathcal{X}}\right) \otimes \omega_{\mathcal{Z} \mid \mathcal{X}}^{-1}\right) \cong \mathbf{L} l^{*} \mathcal{G} \otimes \operatorname{Sym}^{s}\left(\mathcal{T}_{\mathcal{Z} \mid \mathcal{X}}\right)\right|_{\mathcal{Z}_{0}} \otimes \omega_{\mathcal{Z} \mid \mathcal{X}}^{-1}\right|_{\mathcal{Z}_{0}} \otimes \pi_{*} \mathcal{O}_{\mathcal{Z}}
$$

One notices that the terms

$$
\left.\operatorname{Sym}^{s}\left(\mathcal{T}_{\mathcal{Z} \mid \mathcal{X}}\right)\right|_{\mathcal{Z}_{0}} \otimes \omega_{\mathcal{Z} \mid \mathcal{X}}^{-1}{\mid \mathcal{Z}_{0}} \otimes \pi_{*} \mathcal{O}_{\mathcal{Z}}
$$

have weights at least $-t_{\mathcal{Z}}$. When the weights of $\mathcal{G}$ are concentrated in $\left(t_{\mathcal{Z}}, \infty\right)$, we get a trivial weight zero component and the desired vanishing. Now, setting $\mathcal{G}=\mathbf{R H o m}_{\mathcal{X}}(\mathcal{E}, \mathcal{F})$ for $\mathcal{E}, \mathcal{F} \in$ ${ }^{\mathrm{W}} \mathrm{F}^{\mathrm{F}}\left(I, \mathcal{Z}_{0}\right)$, we see that our assumption on $I$ exactly implies this.

Lemma 2.22. Assume that the weights of the conormal sheaf of $\mathcal{Z}$ in $\mathcal{X}$ are all strictly negative, and let $t_{\mathcal{Z}}$ be the weight of the relative canonical sheaf $\omega_{\mathcal{Z} \mid \mathcal{X}}$. Set $\mathcal{U}:=\mathcal{X} \backslash \mathcal{Z}$, and let $j: \mathcal{U} \rightarrow \mathcal{X}$ be the inclusion. Then, the functor,

$$
j^{*}:{ }^{\mathrm{F}}\left(I, \mathcal{Z}_{0}\right) \rightarrow \mathrm{D}^{\mathrm{b}}(\operatorname{coh} \mathcal{U})
$$

is essentially surjective whenever $I$ contains a closed interval of length at least $-t_{\mathcal{Z}}-1$.

Proof. We may assume $\mathcal{X} \neq \mathcal{Z}$, and we may reduce to $I$ being an interval of length $-t_{\mathcal{Z}}-1$. To demonstrate essential surjectivity, it suffices to iteratively reduce the weights by forming exact triangles

$$
\mathcal{E}^{\prime} \rightarrow \mathcal{E} \rightarrow \mathcal{T}
$$

where $\mathcal{T}$ is set-theoretically supported on $\mathcal{Z}$ and the weights of $\mathcal{E}^{\prime}$ are concentrated in an interval strictly smaller than $\mathcal{E}$. There is an exact triangle

$$
\tau_{\geqslant b-1} \mathbf{L} i^{*} \mathcal{E} \rightarrow \mathbf{L} i^{*} \mathcal{E} \rightarrow \mathcal{W},
$$

where the weights of $\mathcal{E}$ along $\mathcal{Z}$ are concentrated in $[a, b]$ with $b-a \geqslant-t_{\mathcal{Z}}$. By Lemma 2.11, the weights of $\tau_{\geqslant b-1} \mathbf{L} i^{*} \mathcal{E}$ are concentrated in $[a, b-1]$. Then, the only weight of $\mathcal{W}$ is $b$. Consider $i_{*} \mathcal{W}$ as an object of $\mathrm{D}^{\mathrm{b}}(\operatorname{coh} \mathcal{X})$. Let us check that the weights of $i_{*} \mathcal{W}$ lie in $\left[b+t_{\mathcal{Z}}, b\right]$. We must compute

$$
\mathbf{L} i^{*} i_{*} \mathcal{W} .
$$

This has cohomology sheaves isomorphic to $\mathcal{W} \otimes \bigwedge^{*}\left(\Omega_{\mathcal{Z} \mid \mathcal{X}}\right)$, which by assumption has weights in $\left[b+t_{\mathcal{Z}}, b\right]$. Note that the only contribution to weight $b$ is $\mathcal{W}$ itself and that the map

$$
\left.\left.\mathbf{L} i^{*} \mathcal{E}\right|_{X_{f}} \rightarrow \mathcal{W}\right|_{X_{f}}
$$




\section{WALL CROSSING FOR DERIVED CATEGORIES}

induces an isomorphism on the weight $b$ portion of the decomposition. Now, we define $\mathcal{E}^{\prime}$ to be the cone over the map $\mathcal{E} \rightarrow i_{*} \mathcal{W}$. Restricting the exact triangle to $X_{f}$ and remembering that computing weight spaces is an exact functor, we see that the weights of $\mathcal{E}^{\prime}$ along $\mathcal{Z}$ are concentrated in $[a, b-1]$. We may also raise the weights by conjugating the previous procedure by dualization. Combining the two procedures, we can move the weights of any complex into $I$ up to $\mathcal{Z}$-torsion.

Corollary 2.23. Assume that the weights of the conormal sheaf of $\mathcal{Z}$ in $\mathcal{X}$ are all strictly negative, and let $t_{\mathcal{Z}}$ be the weight of the relative canonical sheaf $\omega_{\mathcal{Z} \mid \mathcal{X}}$. Set $\mathcal{U}:=\mathcal{X} \backslash \mathcal{Z}$, and let $j: \mathcal{U} \rightarrow \mathcal{X}$ be the inclusion. Then, the functor

$$
j^{*}:{ }^{\mathrm{W}}=\left(I, \mathcal{Z}_{0}\right) \rightarrow \mathrm{D}^{\mathrm{b}}(\operatorname{coh} \mathcal{U})
$$

is an equivalence whenever $I$ is an interval of length $-t_{\mathcal{Z}}-1$.

Proof. This is an immediate consequence of Lemmas 2.21 and 2.22.

Definition 2.24. Let $s \in \mathbb{Z}$. Denote by $\mathcal{C}_{s}(\mathcal{Z})$ the full subcategory of $\mathrm{D}^{\mathrm{b}}\left(\operatorname{coh} \mathcal{Z}_{0}\right)$ consisting of objects with weight $s$.

Lemma 2.25. Assume that the weights of the conormal sheaf of $\mathcal{Z}$ in $\mathcal{X}$ are all strictly negative. The functor

$$
\begin{aligned}
\Upsilon_{s}: \mathcal{C}_{s}(\mathcal{Z}) & \rightarrow \mathrm{D}^{\mathrm{b}}(\operatorname{coh} \mathcal{X}) \\
\mathcal{E} & \mapsto i_{*} \pi^{*} \mathcal{E}
\end{aligned}
$$

is fully faithful.

Proof. We use the standard adjunctions. We have

$$
\operatorname{Hom}_{\mathcal{X}}\left(\Upsilon_{s} \mathcal{E}, \Upsilon_{s} \mathcal{F}\right) \cong \operatorname{Hom}_{\mathcal{Z}}\left(\mathbf{L} i^{*} i_{*} \pi^{*} \mathcal{E}, \pi^{*} \mathcal{F}\right) \text {. }
$$

The cohomology sheaves of $\mathbf{L} i^{*} i_{*} \pi^{*} \mathcal{E}$ are isomorphic to $\pi^{*} \mathcal{E} \otimes \operatorname{Sym}^{*}\left(\mathcal{T}_{\mathcal{Z} \mid \mathcal{X}}\right)$. Thus, there is a natural map

$$
\operatorname{Hom}_{\mathcal{Z}}\left(\pi^{*} \mathcal{E}, \pi^{*} \mathcal{F}\right) \rightarrow \operatorname{Hom}_{\mathcal{X}}\left(\Upsilon_{s} \mathcal{E}, \Upsilon_{s} \mathcal{F}\right)
$$

We first check that this is isomorphism. The cone of $\mathbf{L} i^{*} i_{*} \pi^{*} \mathcal{E} \rightarrow \pi^{*} \mathcal{E}$ has cohomology sheaves $\pi^{*} \mathcal{E} \otimes \operatorname{Sym}^{\geqslant 1}\left(\Omega_{\mathcal{Z} \mid \mathcal{X}}\right)$, which have weights less than $s$. Let us show that

$$
\operatorname{Hom}_{\mathcal{Z}}\left(\pi^{*} \mathcal{E} \otimes \operatorname{Sym}^{\geqslant 1}\left(\Omega_{\mathcal{Z} \mid \mathcal{X}}\right), \pi^{*} \mathcal{F}[s]\right)=0
$$

for any $s$. This vanishing combined with a spectral sequence argument gives us that the map in equation (2.2) is an isomorphism. We have

$$
\begin{aligned}
\operatorname{Hom}_{\mathcal{Z}}\left(\pi^{*} \mathcal{E} \otimes \operatorname{Sym}^{\geqslant 1}\left(\Omega_{\mathcal{Z} \mid \mathcal{X}}\right), \pi^{*} \mathcal{F}[s]\right) & \cong \operatorname{Hom}_{\mathcal{Z}_{0}}\left(\mathcal{E} \otimes \operatorname{Sym}^{\geqslant 1}\left(\Omega_{\mathcal{Z} \mid \mathcal{X}}\right), \mathcal{F} \otimes \operatorname{Sym}^{*}\left(\Omega_{\mathcal{Z}_{0} \mid \mathcal{Z}}\right)[s]\right) \\
& \cong \operatorname{Hom}_{\mathcal{Z}_{0}}\left(\mathcal{E}, \mathcal{F} \otimes \operatorname{Sym}^{*}\left(\Omega_{\mathcal{Z}_{0} \mid \mathcal{Z}}\right) \otimes \operatorname{Sym}^{\geqslant 1}\left(\mathcal{T}_{\mathcal{Z} \mid \mathcal{X}}\right)[s]\right) .
\end{aligned}
$$

We can again factor through a pushforward to the rigidification $\mathcal{Z}_{0}^{\mathbb{G}_{m}}$. In this case, the above Hom-space is zero as the weights of the right-hand side are concentrated in $(s, \infty)$.

Next, we have

$$
\operatorname{Hom}_{\mathcal{Z}}\left(\pi^{*} \mathcal{E}, \pi^{*} \mathcal{F}\right) \cong \operatorname{Hom}_{\mathcal{Z}_{0}}\left(\mathcal{E}, \mathcal{F} \otimes \operatorname{Sym}^{*}\left(\Omega_{\mathcal{Z}_{0} \mid \mathcal{Z}}\right)\right)
$$

The only piece of $\mathcal{F} \otimes \operatorname{Sym}^{*}\left(\Omega_{\mathcal{Z}_{0} \mid \mathcal{Z}}\right)$ in weight $s$ is $\mathcal{F}$. Thus,

$$
\operatorname{Hom}_{\mathcal{Z}_{0}}\left(\mathcal{E}, \mathcal{F} \otimes \operatorname{Sym}^{*}\left(\Omega_{\mathcal{Z}_{0} \mid \mathcal{Z}}\right)\right) \cong \operatorname{Hom}_{\mathcal{Z}_{0}}(\mathcal{E}, \mathcal{F}),
$$

which gives fullness and faithfulness. 


\section{R. BALLARD}

Lemma 2.26. Assume that the weights of the conormal sheaf of $\mathcal{Z}$ in $\mathcal{X}$ are all strictly negative, and let $t_{\mathcal{Z}}$ be the weight of the relative canonical sheaf $\omega_{\mathcal{Z} \mid \mathcal{X}}$. Assume $v-u \geqslant-t_{\mathcal{Z}}$. There is a semi-orthogonal decomposition

$$
{ }^{\mathrm{W}} \mathrm{W}^{\mathrm{F}}\left([u, v], \mathcal{Z}_{0}\right)=\left\langle\Upsilon_{v},{ }^{\mathrm{W}} \mathrm{W}^{\mathrm{F}}\left([u, v-1], \mathcal{Z}_{0}\right)\right\rangle .
$$

Proof. Take $\mathcal{E} \in{ }^{\mathrm{W}^{F}}\left([u, v], \mathcal{Z}_{0}\right)$ and consider the exact triangle from the proof of Lemma 2.22,

$$
\mathcal{E}^{\prime} \rightarrow \mathcal{E} \rightarrow i_{*} \mathcal{W}
$$

It is clear from the definition that $i_{*} \mathcal{W}$ lies in the image of $\Upsilon_{v}$, and we have already seen that $\mathcal{E}^{\prime}$ lies in ${ }^{F}{ }^{F}\left([u, v-1], \mathcal{Z}_{0}\right)$. Thus, the two subcategories generate ${ }^{W^{F}}\left([u, v], \mathcal{Z}_{0}\right)$. It remains to check the semi-orthogonality. This follows as in the proof of Lemma 2.21 .

Remark 2.27. The reader should observe that everything still holds if $\mathcal{X}$ is singular but smooth along $\mathcal{Z}$.

DeFinition 2.28. Let $\mathcal{X}$ be a smooth algebraic stack of finite type over $k$. A stacky BB stratum $\mathcal{Z}$ in $\mathcal{X}$ is called an elementary stratum if the weights of $\Omega_{\mathcal{Z} \mid \mathcal{X}}$ along $\mathcal{Z}$ are strictly negative.

A pair of elementary strata $\mathcal{Z}_{-}$and $\mathcal{Z}_{+}$is called an elementary wall crossing if $\mathcal{Z}_{-, 0}=\mathcal{Z}_{+, 0}$ and the two embeddings of $\mathbb{G}_{m}$ into the automorphisms of $\mathcal{Z}_{ \pm, 0}$ differ by inversion.

Theorem 2.29. Assume that we have an elementary wall crossing given by $\mathcal{Z}_{-}$and $\mathcal{Z}_{+}$. Fix $d \in \mathbb{Z}$.

a) If $t_{\mathcal{Z}_{+}}<t_{\mathcal{Z}_{-}}$, then there are fully faithful functors

$$
\Phi_{d}^{+}: \mathrm{D}^{\mathrm{b}}\left(\operatorname{coh} \mathcal{U}_{-}\right) \rightarrow \mathrm{D}^{\mathrm{b}}\left(\operatorname{coh} \mathcal{U}_{+}\right)
$$

and

$$
\widetilde{\Upsilon}_{j}^{-}: \mathcal{C}_{j}\left(\mathcal{Z}_{-}\right) \rightarrow \mathrm{D}^{\mathrm{b}}\left(\operatorname{coh} \mathcal{U}_{+}\right)
$$

for $-t_{\mathcal{Z}_{-}}+d \leqslant j \leqslant-t_{\mathcal{Z}_{+}}+d-1$ and a semi-orthogonal decomposition

$$
\mathrm{D}^{\mathrm{b}}\left(\operatorname{coh} \mathcal{U}_{+}\right)=\left\langle\widetilde{\Upsilon}_{-t_{\mathcal{Z}_{-}+d}}^{-}, \ldots, \widetilde{\Upsilon}_{-t_{\mathcal{Z}_{+}+d-1}}^{-}, \Phi_{d}^{+}\right\rangle
$$

b) If $t_{\mathcal{Z}_{+}}=t_{\mathcal{Z}_{-}}$, then there is an exact equivalence

$$
\Phi_{d}^{+}: \mathrm{D}^{\mathrm{b}}\left(\operatorname{coh} \mathcal{U}_{-}\right) \rightarrow \mathrm{D}^{\mathrm{b}}\left(\operatorname{coh} \mathcal{U}_{+}\right) .
$$

c) If $t_{\mathcal{Z}_{+}}>t_{\mathcal{Z}_{-}}$, then there are fully faithful functors

$$
\Phi_{d}^{-}: \mathrm{D}^{\mathrm{b}}\left(\operatorname{coh} \mathcal{U}_{+}\right) \rightarrow \mathrm{D}^{\mathrm{b}}\left(\operatorname{coh} \mathcal{U}_{-}\right)
$$

and

$$
\widetilde{\Upsilon}_{j}^{+}: \mathcal{C}_{j}\left(\mathcal{Z}_{+}\right) \rightarrow \mathrm{D}^{\mathrm{b}}\left(\operatorname{coh} \mathcal{U}_{-}\right)
$$

for $-t_{\mathcal{Z}_{+}}+d \leqslant j \leqslant-t_{\mathcal{Z}_{-}}+d-1$ and a semi-orthogonal decomposition

$$
\mathrm{D}^{\mathrm{b}}\left(\operatorname{coh} \mathcal{U}_{-}\right)=\left\langle\widetilde{\Upsilon}_{-t_{\mathcal{Z}_{+}+d}}^{+}, \ldots, \widetilde{\Upsilon}_{-t_{\mathcal{Z}_{-}+d-1}}^{+}, \Phi_{d}^{-}\right\rangle
$$

Proof. The argument is the same as that for the proof of [BFK16, Theorem 3.5.2]. Again, we recall it in some detail. Swapping the roles of $\mathcal{Z}_{+}$and $\mathcal{Z}_{-}$, we can assume $t_{\mathcal{Z}_{+}} \leqslant t_{\mathcal{Z}_{-}}$. Choose intervals $I_{-} \subseteq I_{+}$with the diameter of $I_{ \pm}$equal to $-t_{\mathcal{Z}_{ \pm}}-1$, and let $d:=\min I_{-}=\min I_{+}$. From Lemma 2.26, there is a semi-orthogonal decomposition

$$
\mathrm{WW}^{\mathcal{F}}\left(I_{+}, \mathcal{Z}_{0}\right)=\left\langle\Upsilon_{-t_{\mathcal{Z}_{-}+d}}^{-}, \ldots, \Upsilon_{-t_{\mathcal{Z}_{+}}+d-1}^{-}, \mathrm{WW}^{\mp}\left(I_{-}, \mathcal{Z}_{0}\right)\right\rangle .
$$




\section{WALL CROSSING FOR DERIVED CATEGORIES}

Using Corollary 2.23, we can pull back to $\mathcal{U}_{+}$to get

$$
\mathrm{D}^{\mathrm{b}}\left(\operatorname{coh} \mathcal{U}_{+}\right)=\left\langle i_{+}^{*} \circ \Upsilon_{-t_{\mathcal{Z}_{-}}+d}^{-}, \ldots, i_{+}^{*} \circ \Upsilon_{-t_{\mathcal{Z}_{+}}+d-1}^{-}, i_{+}^{*} \mathrm{~W}^{F}\left(I_{-}, \mathcal{Z}_{0}\right)\right\rangle .
$$

Applying Corollary 2.23 again, we know that $i_{-}^{*}$ induces an equivalence between ${ }^{\mathrm{W}} \mathrm{F}\left(I_{-}, \mathcal{Z}_{0}\right)$ and $\mathrm{D}^{\mathrm{b}}\left(\operatorname{coh} \mathcal{U}_{-}\right)$. To finish, we set

$$
\widetilde{\Upsilon}_{j}^{-}:=i_{+}^{*} \circ \Upsilon_{j}^{-} \text {and } \Phi_{d}^{+}:=i_{+}^{*} \circ\left(i_{-}^{*}\right)^{-1} .
$$

\section{Stable sheaves on rational surfaces}

In this section, we apply Theorem 2.29 using the well-known structure of semi-stable rank two torsion-free sheaves on rational surfaces [EG95, FQ95, MW97].

Let $S$ be a smooth complex projective surface. In this section, we will show how to apply Theorem 2.29 to a wall crossing of Gieseker stable sheaves obtained by variation of the polarization on $S$.

\subsection{Basics}

Let us recall the main notions of stability. Let $L$ be an ample line bundle on $S$.

Definition 3.1. Let $E$ be coherent sheaf on $S$. The sheaf $E$ is Gieseker L-semi-stable [Gie77] if it is torsion free and for any proper subsheaf $F \subsetneq E$, one has $\bar{p}_{L}(F) \leqslant \bar{p}_{L}(E)$, where $\bar{p}_{L}$ is the reduced Hilbert polynomial associated with the embedding given by $L$. If the inequality is strict for any proper subsheaf, $E$ is Gieseker $L$-stable. If $E$ is not Gieseker $L$-semi-stable, then $E$ is Gieseker L-unstable.

The sheaf $E$ is Mumford L-semi-stable [Mum63, Tak72] if it is torsion free and for any proper subsheaf $F \subset E$, one has $\mu_{L}(F) \leqslant \mu_{L}(E)$, where $\mu_{L}$ is the $L$-slope of the sheaf. Again, if the inequality is always strict, $E$ is Mumford $L$-stable and if $E$ is not Mumford $L$-semi-stable, then it is Mumford L-unstable.

Fix invariants $c_{0}, c_{1}, c_{2}$ and consider the moduli functors $\widetilde{\mathcal{M}}_{L}\left(c_{0}, c_{1}, c_{2}\right)$ and $\widetilde{\mathcal{M u m}}_{L}\left(c_{0}, c_{1}, c_{2}\right)$ given by

$X \mapsto\left\{\right.$ iso. classes of Gieseker $L$-s.s. families with $\left.c_{0}\left(\mathcal{F}_{x}\right)=c_{0}, c_{1}\left(\mathcal{F}_{x}\right)=c_{1}, c_{2}\left(\mathcal{F}_{x}\right)=c_{2}\right\}$,

$X \mapsto\left\{\right.$ iso. classes of Mumford $L$-s.s. families with $\left.c_{0}\left(\mathcal{F}_{x}\right)=c_{0}, c_{1}\left(\mathcal{F}_{x}\right)=c_{1}, c_{2}\left(\mathcal{F}_{x}\right)=c_{2}\right\}$.

The following is well known.

Lemma 3.2. The functor $\widetilde{\mathcal{M}}_{L}\left(c_{0}, c_{1}, c_{2}\right)$ is an algebraic stack of finite type over $k$. The same is true for $\widetilde{\mathcal{M u m}}_{L}\left(c_{0}, c_{1}, c_{2}\right)$.

Proof. First, $\widetilde{\mathcal{M}}_{L}\left(c_{0}, c_{1}, c_{2}\right)$ is open [HL10, Proposition 2.3.1] in the stack of coherent sheaves on $S$, which is algebraic [SP16, Theorem 75.5.12]. Thus, $\widetilde{\mathcal{M}}_{L}\left(c_{0}, c_{1}, c_{2}\right)$ is algebraic stack. There is a bounded family of $L$-semi-stable sheaves with fixed numerical invariants [HL10, Theorem 3.3.7]. Base changing the induced map to $\widetilde{\mathcal{M}}_{L}\left(c_{0}, c_{1}, c_{2}\right)$ gives a smooth, surjective map with source of finite type. A similar argument shows the statement for $\widetilde{\mathcal{M u m}}_{L}\left(c_{0}, c_{1}, c_{2}\right)$.

Next, we address smoothness. Recall that $\mathcal{E}$ is Gieseker (Mumford) polystable if it is the direct sum of Gieseker (Mumford) stable sheaves. 


\section{R. BALLARD}

Lemma 3.3. Assume $K_{S}<0$. Let $E$ be Gieseker L-polystable or simple. Then, $E$ is a smooth point of $\widetilde{\mathcal{M}}_{L}\left(c_{0}, c_{1}, c_{2}\right)$. A similar statement holds for $\widehat{\mathcal{M u m}}_{L}\left(c_{0}, c_{1}, c_{2}\right)$.

Proof. Write $E=\bigoplus_{i \in I} E_{i}$ with each $E_{i}$ stable. First, since each $E_{i}$ is stable, or since $E$ is simple, and $S$ is proper, we have

$$
\operatorname{Hom}(E, E) \subset \mathrm{M}_{m}\left(\Gamma\left(S, \mathcal{O}_{S}\right)\right)=M_{m}(k),
$$

where $M_{m}(k)$ is the group of $m \times m$-matrices in $k$.

To check smoothness, it suffices to show that $\operatorname{ext}^{1}(E, E)-\operatorname{hom}(E, E)=\chi\left(\operatorname{Ext}^{*}(E, E)\right)$, which remains constant over $\mathcal{M}_{L}\left(c_{0}, c_{1}, c_{2}\right)$. It suffices, therefore, to show that $\operatorname{Ext}^{2}(E, E)=0$. From Serre duality, we have

$$
\operatorname{ext}^{2}(E, E)=\mathrm{h}^{0}\left(\mathcal{H o m}(E, E) \otimes \omega_{S}\right) .
$$

Since $K_{S}<0$, there exist an inclusion $\omega_{S} \rightarrow \mathcal{O}_{S}$ and an inclusion

$$
\mathcal{H o m}(E, E) \otimes \omega_{S} \rightarrow \mathcal{H o m}(E, E) .
$$

Taking global sections, we see that $\mathrm{h}^{0}\left(\mathcal{H} \mathrm{om}(E, E) \otimes \omega_{S}\right)$ gives the dimension of the subspace of global sections of $\operatorname{Hom}(E, E)$ which vanish along $-K_{S}$. Since all global sections are constant over $S$, we have

$$
\mathrm{h}^{0}\left(\mathcal{H o m}(E, E) \otimes \omega_{S}\right)=0 .
$$

Since Mumford stability implies Gieseker stability, we get the same statement for Mumford semi-stable sheaves.

\subsection{Rank two stable sheaves}

We now restrict ourselves to the case where $S$ is a rational surface and $c_{0}=2$. For notational convenience, we now denote the divisor $c_{1}$ by $\Delta$ and set $c:=c_{2}$. We recall the results of Friedman and Qin [FQ95]; see also [EG95, MW97]. Let $L_{+}$and $L_{-}$be two ample line bundles on $S$. For $\xi \in H^{2}(S, \mathbb{Z})$ satisfying $\xi \equiv \Delta(\bmod 2)$ and $\Delta^{2}-4 c \leqslant \xi^{2} \leqslant 0$, consider the hyperplane in $\operatorname{Amp}(S)_{\mathbb{R}}$ given by

$$
W^{\xi}:=\{D \mid D \cdot \xi=0\} .
$$

The hyperplane $W^{\xi}$ is called the wall associated with $\xi$. For simplicity, we shall assume that the line segment joining $L_{+}$and $L_{-}$intersects only a single $W^{\xi}$, determined by a unique $\xi$. The more general case, where rational multiples of $\xi$ may remain integral and define the same wall, requires minimal modification of the argument [FQ95]. Denote the polarization given by the intersection of the line and the hyperplane by $L_{0}$. We assume that $L_{0}$ lies in no other walls.

We have two inclusions

$$
\widetilde{\mathcal{M}}_{L_{-}}(\Delta, c) \subseteq \widetilde{\mathcal{M u m}}_{L_{0}}(\Delta, c) \supseteq \widetilde{\mathcal{M}}_{L_{+}}(\Delta, c),
$$

where we change the notation

$$
\widetilde{\mathcal{M}}_{L}(2, \Delta, c)=: \widetilde{\mathcal{M}}_{L}(\Delta, c)
$$

to reflect that the focus of our attention is upon rank two sheaves. Note the switch to Mumford semi-stable in the wall. Friedman and Qin study Mumford $L_{0}$-semi-stable sheaves of a particular form.

Definition 3.4. For a divisor $F$ on $S$, let $Z^{k}(F)$ be the set of sheaves $E$ occurring in a short exact sequence

$$
0 \rightarrow I_{Z_{1}}(F) \rightarrow E \rightarrow I_{Z_{2}}(\Delta-F) \rightarrow 0
$$




\section{WALL CROSSING FOR DERIVED CATEGORIES}

where $\xi=2 F-\Delta, Z_{1} \in \operatorname{Hilb}^{k}(S)$, and $Z_{2} \in \operatorname{Hilb}^{l_{\xi}-k}(S)$ with

$$
l_{\xi}=\left(4 c-\Delta^{2}+\xi^{2}\right) / 4 \text {. }
$$

Denote the associated substack of $\widetilde{\mathcal{M u m}}_{L_{0}}(\Delta, c)$ by $\widetilde{\mathcal{Z}}^{k}(F)$.

Proposition 3.5. The substack $\widetilde{\mathcal{Z}}^{k}(F)$ is closed in $\widetilde{\mathcal{M u m}}_{L_{0}}(\Delta, c)$, and $\widetilde{\mathcal{M u m}}_{L_{0}}(\Delta, c)$ is smooth along $\widetilde{\mathcal{Z}}^{k}(F)$.

Proof. A Mumford $L_{0}$-semi-stable sheaf $E$ lies in $Z^{k}(F)$ if and only if there is a surjective map

$$
E(-F) \rightarrow I_{Z_{2}} \rightarrow 0
$$

with $\xi=2 F-\Delta$ and $l\left(Z_{2}\right)=l_{\xi}-k$. This is a closed condition as it states that $E(-F)$ lies in the Quot scheme for the Hilbert polynomial associated with $I_{Z_{2}}$.

By [FQ95, Lemma 2.2], the non-split extensions in $Z^{k}(F)$ are simple. The split extensions are polystable. So Lemma 3.3 gives the last statement.

Applying this proposition with the switch $F \rightarrow \Delta-F$ also gives the corresponding statement for $\widetilde{\mathcal{Z}}^{k}(\Delta-F)$.

The stacks $\widetilde{\mathcal{Z}}^{k}(F)$ admit particularly simple geometric descriptions. Let $\mathcal{E}^{k}(F)$ be the coherent sheaf on $H^{k}:=\operatorname{Hilb}^{k}(S) \times \operatorname{Hilb}^{l_{\xi}-k}(S)$ classifying the extensions appearing in $Z^{k}(F)$; that is, its fiber over $\left(Z_{1}, Z_{2}\right)$ is

$$
\operatorname{Ext}_{S}^{1}\left(I_{Z_{2}}(\Delta-F), I_{Z_{1}}(F)\right) .
$$

By [FQ95, Lemma 2.6], the sheaf $\mathcal{E}^{k}(F)$ is locally free. Let

$$
X^{k}(F):=\underline{\operatorname{Spec}}\left(\mathcal{E}^{k}(F)\right)
$$

be the associated geometric vector bundle on $H^{k}$. There is a natural action of $\mathbb{G}_{m}^{2}$ on $X^{k}(F)$ given by endomorphisms of $I_{Z_{1}}(F) \oplus I_{Z_{2}}(\Delta-F)$, which on a fiber is simply $\left(\alpha \cdot 1_{I_{Z_{1}}(F)}, \beta \cdot 1_{I_{Z_{2}}(\Delta-F)}\right) \cdot e=$ $\alpha e \beta^{-1}=\alpha \beta^{-1} e$ for $e \in \operatorname{Ext}_{S}^{1}\left(I_{Z_{2}}(\Delta-F), I_{Z_{1}}(F)\right)$.

Proposition 3.6. There are isomorphisms

$$
\begin{aligned}
\widetilde{\mathcal{Z}}^{k}(F) & \cong\left[X^{k}(F) / \mathbb{G}_{m}^{2}\right], \\
\widetilde{\mathcal{Z}}^{k}(\Delta-F) & \cong\left[X^{k}(\Delta-F) / \mathbb{G}_{m}^{2}\right] .
\end{aligned}
$$

Proof. From [FQ95, Lemma 2.2.i], given $E$ in $\widetilde{\mathcal{Z}}^{k}(F)$, the schemes $Z_{1}$ and $Z_{2}$ are uniquely determined and the map $\mathcal{I}_{Z_{1}}(F) \rightarrow E$ is unique up to scaling. So the extension class for a given $E$ is determined up to scaling.

To move closer to schemes, we now rigidify our stacks and remove the residual $\mathbb{G}_{m}$ coming from multiples of the identity. We denote the rigidified stacks by removing the tilde; for example, the $\mathbb{G}_{m}$-rigidified moduli stack of Mumford $L_{0}$-semi-stable sheaves will be denoted by $\mathcal{M u m}_{L_{0}}(\Delta, c)$. We do this now, at the current point in the argument, to guarantee that the following result holds.

Proposition 3.7. The substacks $\mathcal{Z}^{k}(F)$ and $\mathcal{Z}^{l_{\xi}-k}(\Delta-F)$ form an elementary wall crossing in the stack $\mathcal{M u m}_{L_{0}}(\Delta, c)$.

Proof. We have a presentation of $\mathcal{Z}^{k}(F)$ given by

$$
\mathbb{G}_{m} \times X^{k}(F)=\mathbb{G}_{m}^{2} / \mathbb{G}_{m} \times X^{k}(F) \rightrightarrows X^{k}(F) .
$$




\section{R. BALLARD}

Now, take the $\mathbb{G}_{m}$ given by the first summand in $\mathbb{G}_{m}$, that is, scalar endomorphisms of $I_{Z_{1}}(F)$. Under this $\mathbb{G}_{m}$-action, $X^{k}(F)$ contracts onto the zero locus, $H^{k}$, so is a BB stratum. This also gives the morphisms $l: \mathbb{G}_{m} \times X^{k}(F) \rightarrow \mathbb{G}_{m}^{2} \times X^{k}(F)$ and $l: \mathbb{G}_{m} \times X^{k}(F) \rightarrow \mathbb{G}_{m}^{2} / \mathbb{G}_{m} \times X^{k}(F)$. The adjoint action is trivial on the first factor and is the action on the second. Thus, it extends to $\mathbb{A}^{1}$ and $\mathcal{Z}^{k}(F)$ is a stacky BB stratum. Computations in [FQ95, Section 3] identify the conormal sheaf of $\mathcal{Z}^{k}(F)$ with $\mathcal{E}^{l_{\xi}-k}(\Delta-F)$, which has weight -1 with respect to this $\mathbb{G}_{m}$-action. So $\mathcal{Z}^{k}(F)$ is an elementary stratum. Similarly, one shows that $\mathcal{Z}^{l_{\xi}-k}(\Delta-F)$ is an elementary stratum. Since we have rigidified, the two choices of $\mathbb{G}_{m}$-actions on the fixed substacks differ by inversion.

From here on, we assume

$$
L_{-} \cdot(2 F-\Delta)<0<L_{+} \cdot(2 F-\Delta)
$$

and

$$
\omega_{S}^{-1} \cdot(2 F-\Delta) \geqslant 0 .
$$

Next, we want to compare the moduli stacks $\mathcal{M}_{L_{+}}(\Delta, c)$ and $\mathcal{M}_{L_{-}}(\Delta, c)$ via a sequence to elementary wall crossings.

To do so, we consider the following intermediate stacks:

$$
\mathcal{M}^{\leqslant l, \geqslant t}:=\operatorname{Mum}_{L_{0}}(\Delta, c) \backslash\left(\bigcup_{k>l} \mathcal{Z}^{k}(F) \cup \bigcup_{k<t} \mathcal{Z}^{k}(\Delta-F)\right) .
$$

Lemma 3.8 ([FQ95, Lemma 3.2.ii]). We have $\mathcal{M}_{L_{+}}(\Delta, c)=\mathcal{M}^{\leqslant l}, \geqslant l_{\xi}+1$ and $\mathcal{M}_{L_{-}}(\Delta, c)=$ $\mathcal{M} \leqslant-1, \geqslant 0$.

We get a sequence of elementary wall crossing given by

$$
\mathcal{M}^{\leqslant l, \geqslant l+1} \subset \mathcal{M}^{\leqslant l+1, \geqslant l+1} \supset \mathcal{M}^{\leqslant l+1, \geqslant l+2} .
$$

Applying Theorem 2.29, we get the following statement.

Proposition 3.9. With the assumptions as above, there is a semi-orthogonal decomposition

$$
\mathrm{D}^{\mathrm{b}}\left(\operatorname{coh} \mathcal{M}^{\leqslant l+1, \geqslant l+2}\right)=\langle\underbrace{\mathrm{D}^{\mathrm{b}}\left(\operatorname{coh} H^{l}\right), \ldots, \mathrm{D}^{\mathrm{b}}\left(\operatorname{coh} H^{l}\right)}_{\mu_{\xi}}, \mathrm{D}^{\mathrm{b}}\left(\operatorname{coh} \mathcal{M}^{\leqslant l, \geqslant l+1}\right)\rangle,
$$

where

$$
H^{l}:=\operatorname{Hilb}^{l}(S) \times \operatorname{Hilb}^{l_{\xi}-l}(S) \quad \text { and } \quad \mu_{\xi}:=\omega_{S}^{-1} \cdot(2 F-\Delta)=\omega_{S}^{-1} \cdot \xi .
$$

Proof. This is an immediate application of Theorem 2.29 using [FQ95, Lemma 2.6] to compute the number of copies of $\mathrm{D}^{\mathrm{b}}\left(\operatorname{coh} H^{l}\right)$.

Corollary 3.10. Let $S$ be a smooth rational surface over $\mathbb{C}$, and let $L_{-}$and $L_{+}$be ample lines bundles on $S$ separated by a single wall defined by the unique divisor $\xi$ satisfying

$$
\begin{gathered}
L_{-} \cdot \xi<0<L_{+} \cdot \xi, \\
0 \leqslant \omega_{S}^{-1} \cdot \xi .
\end{gathered}
$$

Let $\mathcal{M}_{L_{ \pm}}(\Delta, c)$ be the $\mathbb{G}_{m}$-rigidified moduli stack of Gieseker $L_{ \pm}$-semi-stable torsion-free sheaves of rank two with first Chern class $c_{1}$ and second Chern class $c_{2}$. 


\section{WALL CROSSING FOR DERIVED CATEGORIES}

There is a semi-orthogonal decomposition of $\mathrm{D}^{\mathrm{b}}\left(\operatorname{coh} \mathcal{M}_{L_{+}}(\Delta, c)\right)$ as

$$
\langle\underbrace{\mathrm{D}^{\mathrm{b}}\left(\operatorname{coh} H^{l_{\xi}}\right), \ldots, \mathrm{D}^{\mathrm{b}}\left(\operatorname{coh} H^{l_{\xi}}\right)}_{\mu_{\xi}}, \ldots, \underbrace{\mathrm{D}^{\mathrm{b}}\left(\operatorname{coh} H^{0}\right), \ldots, \mathrm{D}^{\mathrm{b}}\left(\operatorname{coh} H^{0}\right)}_{\mu_{\xi}}, \mathrm{D}^{\mathrm{b}}\left(\operatorname{coh} \mathcal{M}_{L_{-}}(\Delta, c)\right)\rangle,
$$

where

$$
l_{\xi}:=\left(4 c_{2}-c_{1}^{2}+\xi^{2}\right) / 4, \quad H^{l}:=\operatorname{Hilb}^{l}(S) \times \operatorname{Hilb}^{l_{\xi}-l}(S), \quad \mu_{\xi}:=\omega_{S}^{-1} \cdot \xi,
$$

with the convention that $\operatorname{Hilb}^{0}(S):=\operatorname{Spec} \mathbb{C}$.

Proof. This is an iterated application of Proposition 3.9 using Lemma 3.8 to identify the first and last moduli spaces.

Remark 3.11. The results on wall crossing of moduli spaces of semi-stable sheaves in [EG95, FQ95, MW97] were originally obtained to compute the change in the Donaldson invariants under a change of the polarization. Thus, Corollary 3.10 can be viewed as a categorification of that wall-crossing formula.

\subsection{An example}

Let us work out the consequences of Corollary 3.10 in the case $S=\mathbb{P}^{1} \times \mathbb{P}^{1}$ with $c_{1}=5 H_{1}+5 H_{2}$ and $c_{2}=14$. We get the following decomposition of the ample cone into walls and chambers:

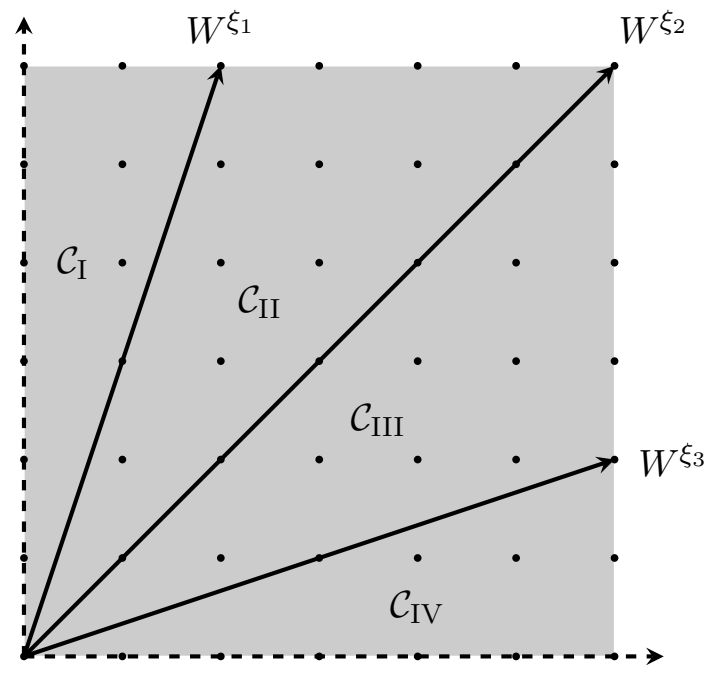

where

$$
\xi_{1}=3 H_{1}-H_{2}, \quad \xi_{2}=H_{1}-H_{2}, \quad \xi_{3}=-H_{1}+3 H_{2} .
$$

We have

$$
l_{\xi_{1}}=0, \mu_{\xi_{1}}=5, \quad l_{\xi_{2}}=1, \mu_{\xi_{2}}=0, \quad l_{\xi_{3}}=0, \mu_{\xi_{3}}=5 .
$$

Let $\mathcal{M}_{J}$ denote the (rigidified) moduli stack of Gieseker $L$-semi-stable torsion-free sheaves of rank two with $c_{1}=5 H_{1}+5 H_{2}$ and $c_{2}=14, L \in \mathcal{C}_{J}$, and $J \in\{$ I, II, III, IV $\}$. Applying Corollary 3.10 to a crossing of $W_{1}$, we see that there is a semi-orthogonal decomposition

$$
\mathrm{D}^{\mathrm{b}}\left(\operatorname{coh} \mathcal{M}_{\mathrm{II}}\right)=\left\langle E_{1}, \ldots, E_{5}, \mathrm{D}^{\mathrm{b}}\left(\operatorname{coh} \mathcal{M}_{\mathrm{I}}\right)\right\rangle
$$




\section{R. BALLARD}

with $E_{i}$ exceptional. Applying it to $W_{2}$, we get an equivalence

$$
\Phi: \quad \mathrm{D}^{\mathrm{b}}\left(\operatorname{coh} \mathcal{M}_{\mathrm{II}}\right) \stackrel{\sim}{\rightarrow} \mathrm{D}^{\mathrm{b}}\left(\operatorname{coh} \mathcal{M}_{\text {III }}\right) .
$$

Applying it to $W_{3}$, we get a semi-orthogonal decomposition

$$
\mathrm{D}^{\mathrm{b}}\left(\operatorname{coh} \mathcal{M}_{\mathrm{III}}\right)=\left\langle F_{1}, \ldots, F_{5}, \mathrm{D}^{\mathrm{b}}\left(\operatorname{coh} \mathcal{M}_{\mathrm{IV}}\right)\right\rangle
$$

with $F_{i}$ exceptional.

The involution $i$ that exchanges the two factors of $\mathbb{P}^{1} \times \mathbb{P}^{1}$ induces isomorphisms

$$
\mathcal{M}_{\mathrm{I}} \cong \mathcal{M}_{\mathrm{IV}} \text { and } \quad \mathcal{M}_{\mathrm{II}} \cong \mathcal{M}_{\mathrm{III}}
$$

so there are really only two moduli spaces here. Note, however, that the equivalence $\Phi$ is not the pullback $i^{*}$ as it leaves unchanged sheaves that are semi-stable in both chambers. Combining the two equivalences, we get an interesting autoequivalence $i^{*} \circ \Phi$ of $\mathrm{D}^{\mathrm{b}}\left(\operatorname{coh} \mathcal{M}_{I I}\right)$ which is a spherical twist around the destabilized locus.

\section{ACKNOWLEDGEMENTS}

The author has benefited immensely from conversations and correspondence with Arend Bayer, Yujiro Kawamata, Colin Diemer, Gabriel Kerr, Ludmil Katzarkov, and Maxim Kontsevich, and would like to thank them all for their time and insight. The author would especially like to thank David Favero for a careful reading of and useful suggestions on a draft of this manuscript. The author is indebted to the referee for generously sharing his time, energy, and perceptive comments. Finally, the author would also like to thank his parents.

\section{REFERENCES}

ABCH13 D. Arcara, A. Bertram, I. Coskun, and J. Huizenga, The minimal model program for the Hilbert scheme of points on $\mathbb{P}^{2}$ and Bridgeland stability, Adv. Math. 235 (2013), 580-626; https: //doi.org/10.1016/j.aim.2012.11.018.

ACV03 D. Abramovich, A. Corti, and A. Vistoli, Twisted bundles and admissible covers, Comm. Algebra 31 (2003), no. 8, 3547-3618; https://doi.org/10.1081/AGB-120022434.

vdBer04 M. van den Bergh, Non-commutative crepant resolutions, in The Legacy of Niels Henrik Abel (Springer, Berlin, 2004), 749-770; https://doi.org/10.1007/978-3-642-18908-1_26.

BFK16 M. Ballard, D. Favero, and L. Katzarkov, Variation of geometric invariant theory quotients and derived categories, J. reine angew. Math., published electronically 13 February 2016, https: //doi.org/10.1515/crelle-2015-0096, to appear in print.

Bia73 A. Białynicki-Birula, Some theorems on actions of algebraic groups, Ann. of Math. 98 (1973), no. 3, 480-497; https://doi.org/10.2307/1970915.

BO95 A. Bondal and D. Orlov, Semi-orthogonal decompositions for algebraic varieties, 1995, arXiv:alg-geom/9506012.

Bri02 T. Bridgeland, Flops and derived categories, Invent. Math. 147 (2002), no. 3, 613-632; https: //doi.org/10.1007/s002220100185.

DS14 W. Donovan and E. Segal, Window shifts, flop equivalences and Grassmannian twists, Compos. Math. 150 (2014), no. 6, 942-978; https://doi.org/10.1112/S0010437X13007641.

EG95 G. Ellingsrud and L. Göttsche, Variation of moduli spaces and Donaldson invariants under change of polarization, J. reine angew. Math. 467 (1995), no. 1, 1-49; https://doi.org/10. 1515/crll.1995.467.1. 


\section{WALL CROSSING FOR DERIVED CATEGORIES}

FQ95 R. Friedman and Z. Qin, Flips of moduli spaces and transition formulas for Donaldson polynomial invariants of rational surfaces, Comm. Anal. Geom. 3 (1995), no. 1-2, 11-83; https://doi.org/10.4310/CAG.1995.v3.n1.a2.

Gie77 D. Gieseker, On the moduli of vector bundles on an algebraic surface, Ann. of Math. 106 (1977), no. 1, 45-60; https://doi.org/10.2307/1971157.

Hal15a D. Halpern-Leistner, The derived category of a GIT quotient, J. Amer. Math. Soc. 28 (2015), no. 3, 871-912; https://doi.org/10.1090/S0894-0347-2014-00815-8.

Hal15b , Remarks on $\theta$-stratifications and derived categories, 2015, arXiv:1502.03083.

HHP08 M. Herbst, K. Hori, and D. Page, Phases of $N=2$ theories in $1+1$ dimensions with boundary, 2008, arXiv:0803.2045; DESY-07-154; CERN-PH-TH/2008-048.

HL10 D. Huybrechts and M. Lehn, The geometry of moduli spaces of sheaves, 2nd ed., Cambridge Math. Lib. (Cambridge Univ. Press, Cambridge, 2010); https://doi.org/10.1017/ CB09780511711985.

HP14 D. Halpern-Leistner and A. Preygel, Mapping stacks and categorical notions of properness, 2014, arXiv:1402.3204.

HW12 M. Herbst and J. Walcher, On the unipotence of autoequivalences of toric complete intersection Calabi-Yau categories, Math. Ann. 353 (2012), no. 3, 783-802; https://doi.org/10.1007/ s00208-011-0704-x.

Kaw02a Y. Kawamata, D-equivalence and K-equivalence, J. Differential Geom. 61 (2002), no. 1, 147171; http://projecteuclid.org/euclid.jdg/1090351323.

Kaw02b _ Francia's flip and derived categories, in Algebraic Geometry (de Gruyter, Berlin, 2002), 197-215.

Kaw06_ Derived categories of toric varieties, Michigan Math. J. 54 (2006), no. 3, 517-535; https://doi.org/10.1307/mmj/1163789913.

Kuz10 A. Kuznetsov, Derived categories of cubic fourfolds, in Cohomological and Geometric Approaches to Rationality Problems, Progr. Math., vol. 282 (Birkhäuser Boston, Inc., Boston, MA, 2010), 219-243; https://doi.org/10.1007/978-0-8176-4934-0_9.

Mum63 D. Mumford, Projective invariants of projective structures and applications, Proc. Internat. Congr. Mathematicians (Stockholm, 1962) (Inst. Mittag-Leffler, Djursholm, 1963), 526-530.

MW97 K. Matsuki and R. Wentworth, Mumford-Thaddeus principle on the moduli space of vector bundles on an algebraic surface, Internat. J. Math. 8 (1997), no. 1, 97-148; https://doi.org/ 10.1142/S0129167X97000068.

Orl93 D. Orlov, Projective bundles, monoidal transformations, and derived categories of coherent sheaves, Russian Acad. Sci. Izv. Math. 41 (1993), no. 1, 133-141; https://doi.org/10.1070/ IM1993v041n01ABEH002182.

Orl97 _ Equivalences of derived categories and K3 surfaces, J. Math. Sci. 84 (1997), no. 5, 1361-1381; https://doi.org/10.1007/BF02399195.

Orl09_ Derived categories of coherent sheaves and triangulated categories of singularities, in Algebra, Arithmetic, and Geometry: in Honor of Yu.I. Manin, Vol. II, Progr. Math., vol. 270 (Birkhäuser Boston, Inc., Boston, MA, 2009), 503-531; https://doi.org/10.1007/ 978-0-8176-4747-6_16.

Seg11 E. Segal, Equivalence between GIT quotients of Landau-Ginzburg B-models, Comm. Math. Phys. 304 (2011), no. 2, 411-432; https://doi.org/10.1007/s00220-011-1232-y.

Shi12 I. Shipman, A geometric approach to Orlov's theorem, Compos. Math. 148 (2012), no. 5, 13651389; https://doi.org/10.1112/S0010437X12000255.

SP16 The Stacks Project Authors, Stacks Project, version 2016, available at http://stacks.math. columbia.edu.

Tak72 F. Takemoto, Stable vector bundles on algebraic surfaces, Nagoya Math. J. 47 (1972), 29-48, http://projecteuclid.org/euclid.nmj/1118798682. 


\section{R. BALLARD}

Tel00 C. Teleman, The quantization conjecture revisited, Ann. of Math. 152 (2000), no. 1, 1-43; https://doi.org/10.2307/2661378.

Matthew Robert Ballard ballard@math.sc.edu

University of South Carolina, Columbia, SC, USA

Current address: Institute for Advanced Studies, Princeton, NJ, USA 